\title{
A NOTE ON THE EXISTENCE OF G-MAPS BETWEEN SPHERES
}

\author{
STEFAN WANER
}

\begin{abstract}
Let $G$ be a finite group, and let $V$ and $W$ be finite-dimensional real orthogonal $G$-modules with $V \supset W$, and with unit spheres $S(V)$ and $S(W)$ respectively. The purpose of this note is to give necessary sufficient conditions for the existence of a $G$-map $f: S(V) \rightarrow S(W)$ in terms of the Burnside ring of $G$ and its relationship with $V$ and $W$. Note that if $W$ has a nonzero fixed point, such a $G$-map always exists, so for nontriviality, we assume this not the case.
\end{abstract}

Existence of $G$-maps. Let $V$ be a finite-dimensional orthogonal $G$-module and let $W \subset V$ be an invariant sub- $G$-module. Denote the unit spheres of $V$ and $W$ by $S(V)$ and $S(W)$ respectively. Here we obtain an algebraic criterion for the existence of a $G$-map $f: S(V) \rightarrow S(W)$. Thus, for nontriviality, we assume $W^{G}=\{0\}$.

The case $V=W$ has been studied in [3], and we first recall pertinent facts. Let $A(G)$ be the Burnside ring of $G$. Thus, $A(G)$ is the Grothendieck group of equivalence classes of finite $G$-sets with addition given by disjoint union. Its elements are thus represented by virtual $G$-sets, and $A(G)$ is additively the free abelian group with basis $\{G / H\}$, where $H$ runs through representatives of conjugacy classes of subgroups of $G$. The multiplicative structure is given by cartesian product. One has a natural isomorphism

$$
\Phi: A(G) \cong \omega_{G},
$$

where $\omega_{G}$ denotes the zeroth equivariant stable stem. (See, for example, [2]. Roughly, $\Phi$ is defined via the collapse map associated with a suitable embedding of a finite $G$-set in a large sphere $S(V)$.) Denote by $\phi(G)$ the set of conjugacy classes of subgroups of $G$, and let

$$
d: A(G) \rightarrow \prod_{(H) \in \phi(G)} \mathbf{Z}=C
$$

denote its integral closure. Thus $d[s-t]_{(H)}=|s|^{H}-|t|^{H}$ for a virtual $G$-set $s-t$. It is well known that $d$ is a monomorphism [1]. Denote by $\Lambda(W)$ the monoid of (free) $G$-homotopy classes of $G$-maps $S(W) \rightarrow S(W)$, and let $\nu(W): \Lambda(W) \rightarrow A(G)$ denote the natural monoid homomorphisms obtained by suspending and applying $\Phi^{-1}$. The results of [3] give a characterization of the image of $\nu(W)$, which we now state. (The constructions there of $G$-maps $S(W) \rightarrow S(W)$ representing suitable elements in $A(G)$ are given in terms of appropriate tangent $G$-vector fields on $S(W)$.)

Received by the editors September 6, 1985 and, in revised form, December 19, 1985. 1980 Mathematics Subject Classification (1985 Revision). Primary 54H15. 
Proposition. An element $a=[s-t] \in A(G)$ is in the image of $\nu(W)$ iff the following conditions hold on $s-t$.

(i) Recalling that $W^{G}=\{0\}$, one requires that $s-t$ be the form $1+\sum_{i} n_{i} G / H_{i}$, where the $H_{i}$ are isotropy subgroups of points in $W-\{0\}$.

(ii) If $H$ is an isotropy subgroup in $W-\{0\}$ and $\operatorname{dim} W^{H}=1$, then

$$
d(a)_{H}= \begin{cases}1 \text { or }-1 & \text { if } N H \neq H ; \\ 0,1 \text { or }-1 & \text { if } N H=H .\end{cases}
$$

One now has the following

THEOREM. With $V$ and $W$ as above, there exists a G-map $S(V) \rightarrow S(W)$ iff:

(a) For each $H \subset G, \operatorname{dim} V^{H} \geqslant 1$ implies $\operatorname{dim} W^{H} \geqslant 1$.

(b) There exists $a \in A(G)$ of the form $1+\sum_{i} n_{i} G / H_{i}$ with each $H_{i}$ an isotropy subgroup of $W$ such that

(i) $d(a)_{H}=0$ whenever $\operatorname{dim} V^{H}>\operatorname{dim} W^{H}$;

(ii) if $\operatorname{dim} V^{H}=1$, then

$$
d(a)_{H}= \begin{cases}1 \text { or }-1 & \text { if } N H \neq H \\ 0,1 \text { or }-1 & \text { if } N H=H .\end{cases}
$$

(Note that $(\mathrm{b})$ is equivalent to the following assertion:

(b)' There exists an element $a \in \operatorname{Im} \nu(W)$ with $d(a)_{H}=0$ whenever $\operatorname{dim} V^{H}>$ $\operatorname{dim} W^{H}$.)

Proof. We first show that the conditions are necessary. Condition (a) is clearly necessary, while, given any $G$-map $f: S(V) \rightarrow S(W)$, composing with the inclusion $i: S(W) \rightarrow S(V)$ gives a $G$-map $g$ on $S(W)$ whose fixed set degrees are zero whenever $\operatorname{dim} V^{H}>\operatorname{dim} W^{H}$, and we take $a$ as $\nu(W)(g)$.

Conversely, assume that conditions (a) and (b) hold. Choose $a \in \operatorname{Im} \nu(W)$ satisfying condition (b), and choose a $G$-map $\rho: S(W) \rightarrow S(W)$ with $\nu(W)(\rho)=a$. By condition (a), if $H \subset G$, is such that $\operatorname{dim} V^{H}=1$, then $\operatorname{dim} W^{H}=1$ as well. This, together with condition (a) itself, permits one to define a $G$-map $\lambda_{0}$ from the zero skeleton of $S(V)$ to $S(W)$, with respect to some $G$-CW decomposition of $S(V)$. Thus assume that we have constructed a $G$-map

$$
\lambda_{n}: S(V)^{n} \rightarrow S(W),
$$

where $S(V)^{n}$ denotes the $n$-skeleton of $S(V)$. The obstruction to extending $\lambda_{n}$ over a typical $(n+1)-G$-cell of the form $G / H \times D^{n+1}$ defines an element $x$ of $\pi_{n}\left(S(W)^{H}\right)$. We consider two cases. If $n<\operatorname{dim} W^{H}-1$, then the obstruction vanishes for dimensional reasons, and one may extend over the given $G$-cell. If $n \geqslant \operatorname{dim} W^{H}-1$, then one has $\operatorname{dim} V^{H}>\operatorname{dim} W^{H}$. Let $\lambda_{n}^{\prime}=\rho \circ \lambda_{n}$. Then, since now $\operatorname{deg}\left(\rho^{H}\right)=0$, and since the obstruction to extending $\lambda_{n}^{\prime}$ over the cell is given by $\rho_{*}^{H}(x)=0$, the obstruction now vanishes. Continuing this process inductively now gives the desired result.

Remarks. Conditions (a) and (b) always hold in the following situation. Let $G$ be any nonsolvable group. Then one has, by [1], a nontrivial idempotent $e$ in $A(G)$, and we may assume that $d(e)_{\{1\}}=0$, where $\{1\}$ denotes the trivial subgroup of $G$. 
If $H$ is a minimal subgroup for which $d(e)_{H}=1$, then, if $f: A(G) \rightarrow A(H)$ denotes the forgetful homomorphism (which assigns to any virtual $G$-set the associated $H$-set via restriction), one has, in $A(H), d(f(e))_{K}=0$ for all proper subgroups $K \subset H$, while $d(f(e))_{H}=1$. Let $R$ be the reduced regular representation of $H$, let $W=R$ $\oplus R$, and let $V=W \oplus W$. Then $\nu(W)$ contains all $H$-sets of the form $1+\sum_{i} H / K_{i}$ wiht $K_{i} \subset H$ proper, whence it contains $f(e)$. It follows from the theorem that there is an $H$-map $S(V) \rightarrow S(W)$. This in turn gives a $G$-map $S(i V) \rightarrow S(i W)$, where $i$ denotes induction.

The existence of such $G$-maps is by no means restricted to nonsolvable, or even to nonabelian groups; let $G=\mathbf{Z} / p \times \mathbf{Z} / q$, with $p$ and $q$ distinct primes. Choose integers $m$ and $n$ with $m p+n q=1$, and let $V=\rho_{p} \oplus \rho_{q} \oplus \rho_{p q}, W=\rho_{p} \oplus \rho_{q}$, where $\rho_{p}$ is any one-dimensional irreducible complex $\mathbf{Z} / p$-module, regarded as a $(\mathbf{Z} / p \times \mathbf{Z} / q \cong \mathbf{Z} / p q)$-module via projection, and similarly for $\rho_{q}$ and $\rho_{p q}$. Then $S(V)$ and $S(W)$ possess isomorphic fixed-sets by any nontrivial subgroup, and we may take $a=1-m \mathbf{Z} / p-n \mathbf{Z} / q$ as our element in $A(\mathbf{Z} / p q)$.

We state an easy consequence of the theorem.

Corollary. Let $W \subset V$ be any G-modules with $V^{G}=W^{G}$, and assume that if $H \subset G$ and $V^{H} \neq V^{G}$, then $W^{H} \neq W^{G}$. Denote the orthogonal complement of $V^{G}$ by $V(G)$, and similarly for $W(G)$. Then there exists a G-map $f: S(V) \rightarrow S(W)$ with fixed-set degree prime to $|G|$ iff (b) above holds with $V$ and $W$ there replaced by $V(G)$ and $W(G)$ respectively. (The condition on fixed sets by subgroups may be thought of as a mild "gap hypothesis," and guarantees that (a) holds in this context.)

Proof. Note that if $V^{G}=W^{G}=0$, then this is just a restatement of the theorem. Thus assume $\operatorname{dim} V^{G} \geqslant 1$. Conditions (a) and (b) are certainly sufficient; one may suspend any $G$-map $S^{n} \rightarrow S^{n}$, where $n=\operatorname{dim} V^{G}-1$, with the unreduced suspension of a $G$-map $S(V(G)) \rightarrow S(W(G))$ to obtain a $G$-map of the desired degree. Conversely, given a $G$-map $f: S(V) \rightarrow S(W)$ with fixed-set degree prime to $|G|$, one has, for suitable $m, m \operatorname{deg}\left(f^{G}\right) \equiv 1 \bmod |G|$ (and where we may take $m= \pm 1$ if $\operatorname{dim} V^{G}=1$ ). This in turn gives an element $a \in A(G)$ satisfying condition (b) of the hypothesis of the theorem. Indeed, one obtains, by classical general position arguments, an element $a \in A(G)$ which represents the $G$-map $m f \circ$ (inclusion of $S(W)$ in $S(V)$ ). The crucial point here is that, since $V^{G} \neq 0$, general position arguments work, since we have a stationary basepoint to map into. Observing that any representing virtual $G$-set has orbit-types those of $W$, and that if $H \neq G$ is any isotropy subgroup occurring in $W$, one has $\operatorname{dim} W^{H} \neq 1$, one now sees that $a \in$ $\nu(W(G))$ and satisfies the conditions (a) and (b) of the theorem.

\section{REFERENCES}

1. T. tom Dieck, Transformation groups and representation theory, Lecture Notes in Math., vol. 766, Springer-Verlag, Berlin and New York, 1979.

2. G. Segal, Equivariant stable homotopy theory, Proceedings ICM, Nice, 1970.

$3 \mathrm{~S}$. Waner and $\mathrm{Y}$. Wu, The local structure of tangent G-vector fields, Topology Appl. (to appear).

Department of Mathematics, Hofstra University, Hempstead, New York 11550 